\title{
Managing the cost of cardiovascular prevention in primary care
}

\section{N Evans}

Heart 2004;90(Suppl IV):iv26-iv28. doi: 10.1136/hrt.2004.037598

Prescribing costs for the prevention of cardiovascular disease are rising nationally, particularly in relation to implementation of the National Service Framework for coronary heart disease. Prescribing effective treatment that is going to benefit the patient's overall managementevidence based medicine-is the key to funding drugs for cardiovascular prevention. It is clear that there is a lot of waste within the system. If the current waste in prescribing can be reduced, it should be possible to fund new developments, not only in cardiovascular disease but also in other therapeutic areas.

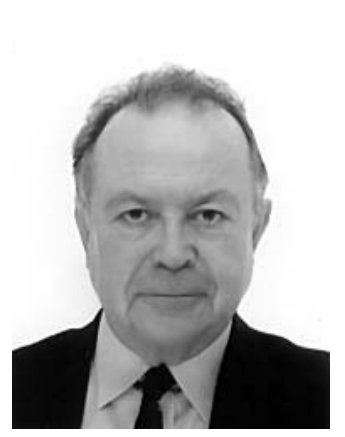

$T$ he Royal College of General Practitioners' guide to good medical practice ${ }^{1}$ describes an "excellent" doctor as one who "only prescribes treatments which make an effective contribution to the patients' overall management" and "takes resources into account when choosing between treatments of similar effectiveness." In contrast, the "unacceptable" doctor is one who "consistently prescribes unnecessary or ineffective treatments" and "takes no note of resources when choosing between similar treatments." Prescribing effective treatment that is going to benefit the patient's overall management is evidence based medicine and this is the key to funding drugs in the field of cardiovascular disease, and all other therapeutic areas.

Some of the variables that are known to affect prescribing are shown in table 1 . The young and the elderly require more medication than other groups. In terms of number of practice partners, there is evidence that single handed doctors are higher cost prescribers than multiple partner doctors. However, the larger the practice, the greater the range of drugs that are being prescribed. ${ }^{2}$ The average doctor prescribes from a range of around 200 drugs, but in a practice of five or more doctors up to 1000 different drugs are probably being prescribed.

Marketing activity is an important factor. Practices that see pharmaceutical industry representatives generally have higher prescribing costs than those that do not. In terms of chronic disease management, there is an issue relating to hospital initiated treatment. In a 1998 survey carried out in Wandsworth on lipid management, only $9 \%$ of patients who had been started on lipid lowering treatment in secondary care were actually at target cholesterol concentrations. Patients were not being reviewed by general practitioners, whereas the hospital SWI7 7DJ, UK; norman. evans@swlondon.nhs.uk doctors thought they had discharged the patients back to primary care.

Repeat prescribing accounts for $80 \%$ of prescribing costs, and this is where waste occurs. In a study reported by McGavock and colleagues ${ }^{3}$ in Northern Ireland, around $40 \%$ of prescribed medicines were not actually taken. For Wandsworth Primary Care Trust (PCT), which has a drugs bill of $£ 30$ million, that equates to $£ 12$ million of wasted medicines. In another study, Bond and colleagues ${ }^{4}$ looked at practices in Aberdeen and identified a waste medicine bill of $£ 43$ per patient per year. Reducing this waste, which involves paying attention to patient compliance, is the key to enabling the health service to pay for new medicines. The money is already in the system, if a way can be found to access it.

Cardiovascular prescribing costs for 2002 in England are shown in table 2. Total prescribing costs were $£ 6.84$ billion and about one quarter of that ( $£ 1.7$ billion) related to cardiovascular drugs. ${ }^{5}$ The highest proportion of this was accounted for by angiotensin converting enzyme (ACE) inhibitors and angiotensin receptor blockers, followed by calcium channel blockers. However, felodipine and amlodipine (the leading calcium channel blockers in the UK) will soon lose patent protection, which may offer potential for cost savings.

\section{Wandsworth activities}

Prescribing costs in relation to the prevention of coronary heart disease (CHD) are increasing nationally, with significant cost pressures related to implementation of the National Service Framework (NSF) for CHD. As part of Wandsworth PCT'S response to the NSF, a mapping exercise was carried out to find out what was happening in each practice in the three localities that make up the PCT. The aim was to identify which elements of the NSF were already being monitored and whether this was occurring in a uniform way across the PCT. From this, decisions could be taken on how the PCT should respond to the NSF and the implications for prescribing.

The mapping exercise identified systems and processes-for example, information was collected on the proportion of patients who were currently being monitored, how many people had cholesterol values above $5 \mathrm{mmol} / \mathrm{l}$, whether smoking status had been recorded, and whether

\footnotetext{
Abbreviations: $A C E$, angiotensin converting enzyme; ALLHAT, antihypertensive and lipid lowering treatment to prevent heart attack trial; CHD, coronary heart disease; GMS, General Medical Service; NSF, National Service Framework; PCT, primary care trust 
Table 1 Variables affecting prescribing habits

- Population: $<5$ years, $>65$ years

- Number of partners in practice

- Marketing

- Chronic disease (hospital initiated prescribing)

- Repeat prescribing

- Waste/concordance

- Miscellaneous, e.g. single parent, unemployment
Table 3 Primary care trust-wide targets

- Written policy on repeat prescribing

- National Service Framework for older people: medication reviews

- Management of patients with ischaemic heart disease:

$-90 \%$ patients on aspirin or alternative antiplatelet agent unless contraindicated

$-80 \%$ cholesterol measured/recorded and lipid treatment commenced unless contraindicated smokers had been offered smoking cessation advice. Following this data collection, an implementation group was set up and CHD guidelines were established for angina, lipids, and hypertension. Guidelines are currently being developed for stroke/transient ischaemic attack, smoking, physical activity, and healthy eating.

The information from the mapping exercise has also been linked into the development of cardiovascular indicators within the Wandsworth prescribing incentive scheme and to the quality and outcomes framework within the new General Medical Services (GMS) contract.

The prescribing incentive scheme has been crucial in allowing the PCT to contain its prescribing costs Wandsworth PCT is the fourth largest PCT in England, covering a population of 320 000, but it has the ninth lowest prescribing costs. At present, the PCT is significantly under budget.

All practices participate and benefit in this scheme which encourages rational, cost effective prescribing using best available evidence. The scheme also encourages generic prescribing. It could, however, be argued that the importance of generic prescribing is to some extent overplayed: the initial target was $72 \%$ generic prescribing by 2002 ; that target was reached, and the Department of Health has now raised the target and the generic prescribing rate is now almost $80 \%$. Yet since around $80 \%$ of prescribing costs relate to branded products, is it still appropriate to concentrate on increasing generic prescribing? It is probably more cost effective to look at the overall appropriateness of prescribing.

The prescribing incentive scheme has some PCT-wide targets (table 3 ). In addition, practices are offered a choice of 10 other targets, to be chosen in agreement with the locality prescribing adviser. One of these targets, in line with the results of the antihypertensive and lipid-lowering treatment to prevent heart attack trial (ALLHAT), ${ }^{6}$ is to encourage use of thiazides as first line treatment for hypertension and to reduce prescribing of calcium channel blockers by $10 \%$. Another relates to the need to develop and audit prescribing in line with British Hypertension Society guidelines, and to increase the number of patients reaching optimal blood pressure targets (140/85 mm Hg, or 140/ $80 \mathrm{~mm} \mathrm{Hg}$ for diabetics). ${ }^{7}$ The targets do not, however, all relate to cardiovascular prescribing-for example, others cover use of antibiotics, benzodiazepines, non-steroidal

Table 2 Cardiovascular prescribing costs, England, 2002 5

\begin{tabular}{ll}
\hline Total prescribing costs & $£ 6.84$ billion \\
Cardiovascular drug costs & $£ 1.74$ billion \\
Bendrofluazide & $£ 21$ million \\
$\beta$ Blockers & $£ 89$ million \\
Atenolol & $£ 21$ million \\
ACE inhibitors/angiotensin receptor & \\
blockers & $£ 500$ million \\
Calcium channel blockers & $£ 290$ million \\
Amlodipine & $£ 140$ million \\
\hline
\end{tabular}

anti-inflammatory drugs, proton pump inhibitors, and antidepressants.

\section{Future cost pressures}

All PCTs attempt to assess the costs likely to arise over the next two or three years in relation to therapeutic developments. According to the UK Medicines Information database (http://www.ukmi.nhs.uk/Med_info/NewProd.asp), new drugs such as the lipid lowering drug ezetimibe are likely to lead to significant prescribing costs in the cardiovascular arena. Implementing trial evidence on the use of ACE inhibitors for heart failure is estimated to require use of these drugs in around 1500 patients per 100000 population. With an $80 \%$ uptake this is likely to cost around $£ 140000$. On a more positive note, however, it has been calculated that full implementation of the ALLHAT trial could lead to savings of about $£ 125000$ per 100000 population. In addition, earlier use of thrombolysis in acute myocardial infarction, the use of drug eluting stents, and the wider use of drugs such as glycoprotein IIb/IIIa antagonists and clopidogrel will add further pressure.

Furthermore, there is concern over whether or not the new GMS contract has been costed properly. Full implementation of the new quality and outcomes framework could prove to be the major cost pressure on PCTs over the next few years, far outstripping the costs of any new drugs. It has been estimated that costs could increase by between $2.2 \%$ and $5.6 \%$ above inflation. $^{8}$

\section{Evidence based medicine}

Examination of the 20 leading cost drugs in the south west London area leads to consideration of whether prescribers are currently practising evidence based medicine (representing value for money) or whether prescribing is related more to market forces. For example, £3.2 million is currently being spent on simvastatin, the PCT's leading cost drug. Simvastatin is an effective drug, with good supporting evidence, but two thirds of prescriptions are for the $10 \mathrm{mg}$ dose. The Scandinavian simvastatin survival study trial ${ }^{9}$ used an average dose of $27 \mathrm{mg}$ and the heart protection study ${ }^{10}$ used $40 \mathrm{mg}$ of simvastatin, so there is considerable expenditure on suboptimal use of simvastatin.

Losartan is also among the leading cost drugs. While it is an effective drug, it has a relatively flat dose-response curve. Prescribing of losartan has increased by $35 \%$ a year-as PCTs seek to manage their drugs expenditure, they must address the question of whether such use is justified or whether perhaps other drugs in the same class might be more cost effective.

\section{CONCLUSION}

Prescribing costs in relation to cardiovascular prevention are increasing nationally, with particular cost pressures related to implementation of the NSF for CHD. However, if evidence based prescribing can be implemented, and current waste reduced, it should be possible to fund most of the new developments, not only in cardiovascular disease but also in other therapeutic areas. 


\section{REFERENCES}

1 Royal College of General Practitioners/General Practitioners Committee Good medical practice for general practitioners. London: RCGP, 2002.

2 McCarthy M, Wilson-Davis K, McGavock H. Relationship between the number of partners in a general practice and the number of different drugs prescribed by that practice. Br J Gen Pract 1992;354:10-12.

3 McGavock H, Wilson-Davis K, Connolly JP. Repeat prescribing management - a cause for concern? Br J Gen Pract 1999;49:343-7.

4 Bond C, Matheson C, Williams S, et al. Repeat prescribing: a role for community pharmacists in controlling and monitoring repeat prescriptions. Br J Gen Pract 2000:50:271-5.

5 Department of Health. Prescription cost analysis: England 2002. http:// www.doh.gov.uk/stats/pca2002.pdf [accessed 7 December 2003].

6 The ALLHAT Officers and Co-ordinators for the ALLHAT Collaborative Research Group. Major outcomes in high-risk hypertensive patients randomised to angiotensin-converting enzyme inhibitor or calcium channel blocker vs diuretic. The antihypertensive and lipid-lowering treatment to prevent heart attack trial (ALLHAT). JAMA 2002;288:2981-97.

7 Ramsay LE, Williams B, Johnston GD, et al. British Hypertension Society guidelines for hypertension management 1999: summary. BMJ 1999;319:630-5.

8 Prescribing Support Unit. National prescribing costs implications of the new GMS contract. Leeds: Prescribing Support Unit, January 2004.

9 Scandinavian Simvastatin Survival Study Group. Randomised trial of cholesterol lowering in 4444 patients with CHD: the Scandinavian simvastatin survival study (4S). Lancet 1994;344:1383-9.

10 Heart Protection Study Collaborative Group. MRC/BHF heart protection study of cholesterol lowering with simvastatin in 20,536 high-risk individuals: a randomised placebo-controlled trial. Lancet 2002;360:7-22.

\section{DISCUSSION}

Question: Can I ask a "generic" question about the input of pharmacists in cardiovascular prevention in UK. Do you think there is a future for pharmacists in relation to cardiovascular prevention?

Mr Evans: I do think that there is a role for community pharmacists. They see people when they are relatively well, before they get to GPs. However, the concern I have with community pharmacists doing ad-hoc, impromptu screening is that if they do it without adequate training they are going to create the "worried well". I don't believe that items such as cholesterol testing kits, blood glucose meters, etc, should be sold over the counter in pharmacies without adequate training, and without associated health promotion and lifestyle advice. In Wandsworth PCT we have a pharmacist and a nurse running a health promotion clinic in a pharmacy; interestingly, they pick up more diabetes than patients with hypertension or high cholesterol. So I think there is a role for adequately trained community pharmacists in the screening process. The proposed deregulation of simvastatin to a pharmacy medicine will bring interesting challenges for community pharmacists. Will it be available at a sufficient dose and at an affordable price? I am not convinced about the proposals and suggest that there are other medicines which should be considered for change first.

Question: Just echoing what you were saying about effective prescribing. Two practices in our PCT have the same list size, the same number of GPs, and the same prevalence of CHD, and they both have their CHD patients hitting a target cholesterol of less than $5 \mathrm{mmol} / \mathrm{l}$ in $70 \%$ of patients. Yet one practice spends twice as much on statins as the other. I think both practices are probably right in their own sense, but one is practising wider primary prevention than the other.

Mr Evans: We have attempted to look at why different practices prescribe differently and can identify about $40 \%$ of the differences. Interestingly, whether the prescriber is male or female has an effect. Female doctors do not issue as many prescriptions but when they do they are more generous with what they give. Colin Bradley identified about 140 different reasons for issuing a prescription. Perhaps men are more aggressive at giving a prescription to end the consultation, but women are more sympathetic prescribers. That is one of the factors. 